\title{
Síndrome de Sézary: um relato de caso com diagnóstico tardio e rápida progressão tumoral
}

\section{Sézary syndrome: a case report with late diagnosis and fast tumoral progression}

\author{
Renata Cordeiro de Araújo ${ }^{1}$, Luiz Arthur Calheiros Leite ${ }^{2}$, Monica da Silva Oliveira ${ }^{3}$, \\ Jailson Ferreira da Silva ${ }^{4}$, Cláudia Wanderley de Barros Correia ${ }^{5}$, Josemir Belo Santos ${ }^{6}$
}

Araújo RC, Leite LAC, Oliveira MS, Silva JF, Correi CWB, Santos JB. Síndrome de Sézary: um relato de caso com diagnóstico tardio e rápida progressão tumoral / Sézary syndrome: a case report with late diagnosis and fast tumoral progression. Rev Med (São Paulo). 2016 jul.-set.;95(3):142-5.

RESUMO: A síndrome de Sézary é raro linfoma de células T que compromete a pele e outros tecidos, correspondendo a $3 \%$ de todos os linfomas cutâneos. O objetivo deste estudo foi relatar um caso de síndrome de Sézary que evoluiu com lesões tumorais na pele. Paciente de 46 anos, sexo masculino, deu entrada no serviço de Dermatologia da UFPE com pequenas lesões eritematosas, evoluindo com eritrodermia esfoliativa, e hiperqueratose palmoplantar. Apresentava 1,1×10\%/L células de Sézary e foi refratário à quimioterapia, progredindo com múltiplas lesões tumorais na pele. A síndrome de Sézary permanece com prognóstico reservado e curta sobrevida, sendo necessário um diagnóstico precoce.

Descritores: Linfoma cutâneo de células T; Síndrome de Sézary; Prognóstico.

\begin{abstract}
Sézary syndrome is rare kind of T-cell lymphoma that compromises the skin and other tissues and is responsible for $3 \%$ of all cutaneous lymphomas. The aim of this study was to report a case with a rapid tumor progression. A 46-year-old male patient was admitted on Dermatology service at the UFPE with skin lesions. The patient developed exfoliative erythroderma and palmoplantar hyperkeratosis. The blood count showed $1.1 \times 10^{9} / \mathrm{L}$ Sézary cells and the patient rapidly progressed with tumor lesions on the skin. Sézary syndrome remains with poor prognosis and short survival.
\end{abstract}

Keywords: Lymphoma, T-cell, cutaneous; Sézary syndrome; Prognosis.

1. Acadêmica de Medicina, Centro Universitário UniNassau, Recife, PE, Brasil. E-mail: renata_araujo@hotmail.com.

2. Professor Doutor, Serviço de Imunologia e $10^{\mathrm{a}}$ enfermaria, Hospital Universitário Gaffrée e Guinle, Centro de Ciências Biológicas e da Saúde, Universidade Federal do Estado do Rio de Janeiro, Rio de Janeiro, Brasil. E-mail: lahemato@hotmail.com.

3. Médica, Departamento de Dermatologia, Centro de Ciências de Saúde, Universidade Federal de Pernambuco (UFPE), Recife, PE, Brasil. E-mail: monicadoliveira@hotmail.com.

4. Acadêmico de Biomedicina, Universidade Federal de Pernambuco (UFPE), Recife, PE, Brasil. E-mail: jailsonbiomed@gmail.com

5. Médica Assistente do Serviço de Hematologia, Hospital das Clínicas, UFPE, Recife, PE, Brasil. E-mail: claudia.wanderley@uol. com.br.

6. Médico, Professor Adjunto e Chefe do Serviço de Dermatologia, Centro de Ciências de Saúde, Universidade Federal de Pernambuco (UFPE), Recife, PE, Brasil. E-mail: josemirbelo@uol.com.br.

Autor para correspondência: Luiz Arthur Calheiros Leite. Rua Mariz e Barros, 775. CEP: 20270-004 Rio de Janeiro, RJ, Brazil. E-mail: lahemato@hotmail.com. 


\section{INTRODUÇÃO}

$\mathrm{O}$ s linfomas cutâneos de células T (LCCTs) são raros linfomas não-Hodgkin que comprometem primariamente a pele, podendo acometer também linfonodos, sangue e outros órgãos extranodais ${ }^{1,2}$. Os LCCTs e os linfomas cutâneos de células B representam $2 \%$ de todos os linfomas com incidência anual de 0,3 a 1 por 100.000 casos $^{3}$. Dentre os LCCTs os mais comuns são a micose fungóide de curso indolente e a síndrome de Sézary (SS) que é a forma leucêmica, geralmente associada a um curso agressivo ${ }^{4}$.

Clinicamente a SS se caracteriza por eritrodermia exfoliativa e pela presença de linfócitos circulantes com núcleos convolutos. Em estágios iniciais a SS pode apresentar lesões de pele indistinguíveis de outras doenças dermatológicas e deve ser diferenciada da eritrodermia de origem inflamatória, como a psoríase e farmacodermias ${ }^{2}$. O diagnóstico de SS é confirmado através da presença das células de Sézary no sangue periférico. O exame histopatológico associado à imunofenotipagem é essencial para a classificação dos linfomas ${ }^{5,6}$.

Recentemente, além das características clínicas, a International Society for Cutaneous Lymphomas (ISCL), propõe que mais de um destes critérios estejam presentes para o diagnóstico de SS, sendo estes a contagem absoluta de células de Sézary $>1.000 / \mathrm{mm}^{3}$ no sangue periférico; demonstração de anormalidades fenotípicas ou do clone de células T por análise citogenética ou molecular ${ }^{2}$.

O tratamento da SS depende do estágio da doença, existindo uma grande quantidade de terapias disponíveis para os pacientes acometidos com esta síndrome. Dentre os regimes terapêuticos utilizados incluem-se: PUVA, corticóides, radioterapia, poliquimioterapia, bexaroteno oral, monoterapia com interferon (INF) alfa, baixas doses de MTX (metotrexate), vorinostate e novas drogas tais como, inibidores de histona, os anticorpos monoclonais (alemtuzumab, zanolimumab), inibidores de proteasoma (bortezomib), lenalidomida e pralatrexate ${ }^{7}$.

Este estudo teve por objetivo descrever um caso de SS com diagnóstico tardio e rápida progressão tumoral, reforçando a necessidade do diagnóstico precoce e do diferencial dos LCCTs de outras lesões dermatológicas.

\section{RELATO DE CASO}

Paciente masculino, 44 anos, natural de Jaboatão dos Guararapes, Pernambuco, procurou a assistência médica em Março de 2014 com quadro de lesões de pele pruriginosas, descamativas e generalizadas. Relatava história pregressa de quatro anos de lesões de pele tratadas em consultas nos postos de saúde. Não apresentava febre ou perda de peso. Durante o internamento na enfermaria da Dermatologia do Hospital das Clínicas, UFPE foi identificado eritrodermia, queratodermia palmo-plantar e onicodistrofia, mas sem organomegalias ou sinais de turmores na pele (Figura 1). O hemograma evidenciou: leucócitos $6,8 \times 10^{9} / \mathrm{L}$, com predominância de linfócitos com

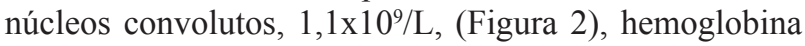
$15,1 \mathrm{~g} / \mathrm{dL}$, plaquetas $235 \times 10^{9} / \mathrm{L}$. Diante disto, o paciente foi avaliado pelo serviço de Hematologia para início da terapia. O exame de MO exibia predomínio de células linfoplasmocitárias ( $56 \%$ de células de Sézary). A LDH foi de 121,8 U/L. (135,0 a 214,0 U/L). A sorologias para os vírus da hepatite $\mathrm{B}$ (HBsAg e anti-HBc), hepatite $\mathrm{C}$ (antiHCV), HIV (anti-HIV) e HTLV (anti-HTLV 1 e 2) foram negativas. A imunofenotipagem por citometria de fluxo demonstrou positividade para os antígenos CD2, CD3, CD4, CD5, clonalidade (TCR $\alpha \beta$ ) e ausência do CD7, CD8 e do CD26. O exame histopatológico de pele evidenciou infiltrado linfóide com epidermotropismo características de linfoma (micose fungóide), (Figura 3 ). Contudo, devido às inúmeras infecções de pele, o paciente recebeu terapia com antibióticos de amplo espectro. Confirmado o diagnóstico de $\mathrm{SS}$ foi iniciado o esquema de poliquímioterapia com CHOP (ciclofosfamida, doxorubicina, vincristina e prednisona). No terceiro ciclo de quimioterapia o paciente apresentou piora clínica, com linfonomegalias axilares e inguinais bilaterais, perda de peso $(8 \mathrm{~kg})$, piora dos nódulos cutâneos (Figura 3), elevação dos níveis séricos de LDH 362,6 U/L e aumento dos leucócitos 57,9×10\%/L, com predominância das células de Sézary $\left(40,1 \times 10^{9} / \mathrm{L}\right)$, sendo encaminhando para realização de PUVA associado com interferon 1500000 U/I semanais. Continuou sem melhora, sendo introduzido MTX em baixas doses semanais e posteriormente monoterapia com gencitabina. $\mathrm{O}$ paciente continuou sem resposta efetiva aos esquemas quimioterápicos, e evoluiu com piora do quadro e progressão rápida da doença, e foi a óbito por sepse.

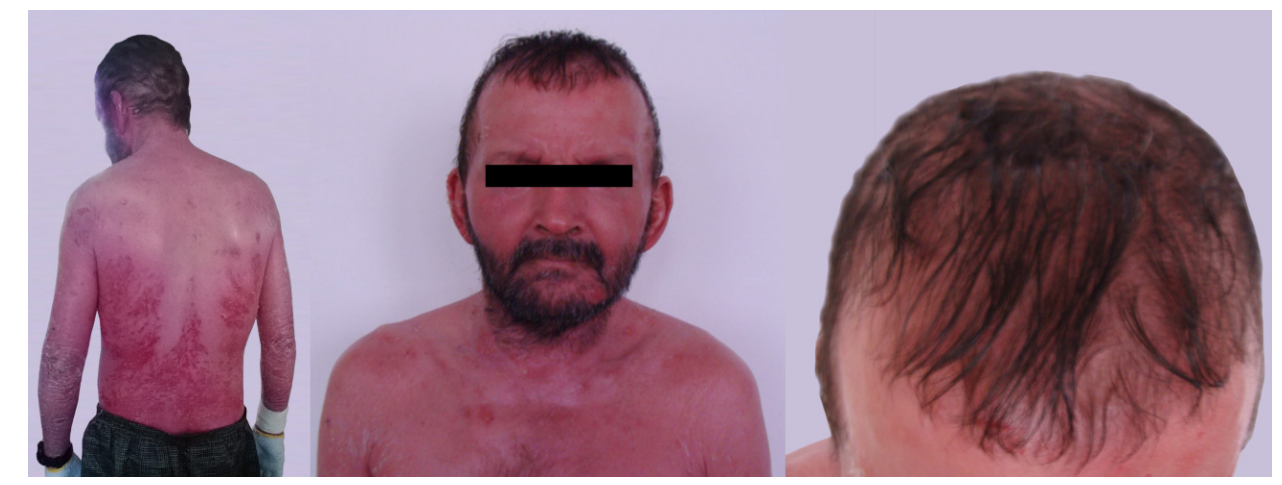

Figura 1. Eritrodermia e marcante alopecia 


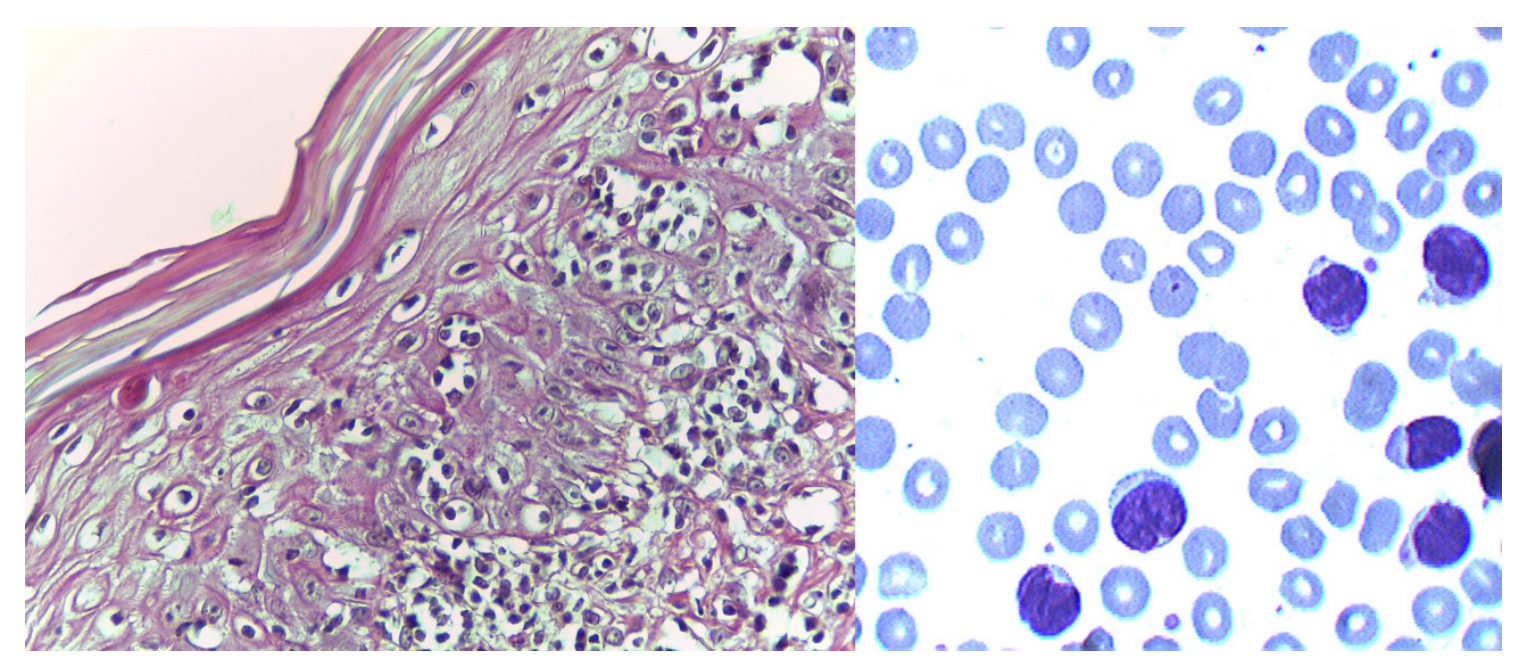

Figura 2. Células de Sézary com núcleos convolutos no exame de sangue periférico (May-Gruwald Giemsa, x1000). Epidermotropismo linfocitário de pele, exame histopatológico (HE, x 400)

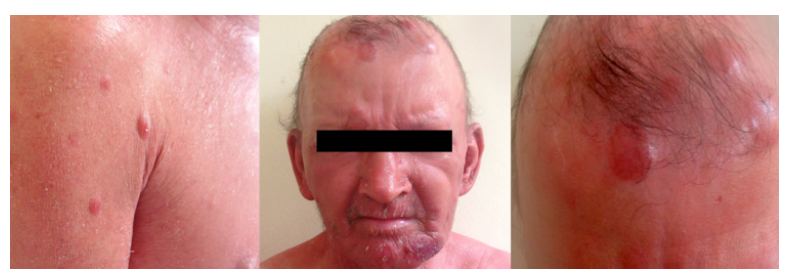

Figura 3. Lesões tumorais em face e membro superior esquerdo

\section{DISCUSSÃO}

A SS é um tipo distinto de linfoma cutâneo de células T eritrodérmico com evidências hematológicas de envolvimento leucêmico, sendo caracterizada por alopecia, hiperqueratose palmo-plantar, acometimento linfonodal e pela presença de linfócitos com núcleos convolutos no exame de sangue periférico ${ }^{3,5}$.

O diagnóstico pode ser difícil, pois tanto os achados clínicos quanto os histológicos podem ser inespecíficos, sugerindo muitas vezes, alterações inflamatórias leves na pele ${ }^{6,8}$. Segundo a WHO-EORTC o diagnóstico de SS deve ser realizado pela presença de pelo menos 1000 células de Sézary por $\mathrm{mm}^{3}$ de sangue periférico com positividade para o CD3, CD4, CD5, CD8 e perda do CD7 e CD26, aumento da população de células T CD4 $4^{+} \mathrm{CD} 7^{-}$e/ou razãoCD4/CD8 $>10$, além da monoclonalidade para o receptor de células T. Estes achados associados a níveis elevados de LDH, devem ser considerados como outros fatores de prognóstico desfavorável, sendo a sobrevida mediana destes pacientes de 31 meses $^{2,9}$.

Em nosso caso, o paciente apresentou inicialmente prurido com pequenas placas vermelhas na pele, dificultando o diagnóstico da SS. Contudo, após o surgimento de eritrodermia com ulcerações, prurido intenso, e principalmente após a visualização de mais de 1100 células de Sézary no sangue periférico, levantou-se a suspeita de SS com posterior confirmação através da biópsia de pele e imunofenotipagem por citometria de fluxo, que confirmou a origem $\mathrm{T}(\mathrm{CD} 2, \mathrm{CD} 3, \mathrm{CD} 4, \mathrm{CD} 5, \mathrm{CCR} 4)$ e ausência do $\mathrm{CD} 7, \mathrm{CD} 8$ e CD26.

O tratamento da SS destina-se a depuração das lesões de pele, minimização da recidiva, prevenção da progressão da doença e preservação da qualidade de vida do paciente. Outras considerações importantes são a gravidade dos sintomas, incluindo prurido e a idade do paciente, além das co-morbidades. Assim, a terapia depende do estádio da doença, no entanto, o tempo de atividade, os sintomas e o grau de infiltração são extremamente variáveis. Nos pacientes com estádios IVA e IVB são adotadas terapias sistêmicas com ou sem tratamentos direcionados à pele. Dentre as opções terapêuticas faz-se uso da fotoforese extracorpórea, modificadores de resposta biológica como o interferons, retinóides e baixas doses de metotrexato. Em casos redicivantes com são usados monoquimioterapia com doxorrubicina lipossomal, gencitabina, análogos da purina ou poliquimioterapia com $\mathrm{CHOP}$ associado ou não com PUVA. Outra linha terapêutica que pode ser adotada utilizando os imunomoduladores, tais como o pralatrexate, lenalidomida, bortezomibe, e os anticorpos monoclonais, alemtuzumabe, mogamulizumab e brentuximab. Contudo o curso clínico da SS é muito agressivo e mesmo com amplo arsenal terapêutico, a SS permanece com prognóstico reservado, com taxas de sobrevida de 5 anos em apenas 10 e 20 dos $\operatorname{casos} \%{ }^{7,10}$.

No presente estudo o tratamento poliquimioterápico não mostrou resposta clínica e o paciente evoluiu rapidamente com piora do quadro e envolvimento tumoral na pele, levando a crer que o clone linfocitário progrediu com disseminação visceral, piorando o prognóstico do paciente. O paciente também apresentou outros dois fatores que condizem com prognóstico desfavorável, como a 
elevação da LDH e o aumento no sangue periférico das células de Sézary para $43 \times 10^{9} / \mathrm{L}$ e evoluiu para óbito em menos de um ano, fato que leva a crer que este paciente apresentou uma doença mais agressiva e com diagnóstico tardio. A abordagem do paciente com SS deve ser feita

\section{REFERÊNCIAS}

1. Mazzeo E, Rubinno L, Buglioni M, Antognoni P, Magrini SM, Bertoni F, Parmiggiani M, Barbieri P, Berton F. The current managment of mycoisfungoides and Sézary syndrome and the role of radiotherapy: principles and indications. Rep Pract Oncol Radiother. 2014;19(2):77-91. doi: 10.1016/j.rpor.2013.07.009.

2. Willenze R, Jaffe ES, Burg G, Cerroni L, Berti E, Swerdlow SH, Ralfkiaer E, Chimenti S, Diaz-Perez JL, Duncan M, Grange F, Harris NL, Kempf W, Kerl H, Kurrer M, Knobler R, Pimpinelli N, Sander C, Santucci M, Sterry W, Vermeer MH, Wechsler J, Whittaker S, Meijer CJ. WHOEORTC classification of cutaneous lymphomas. Blood. 2005;105(10):3768-85. doi: 10.1182/blood-2004-09-3502.

3. Yamashita T, Abbade LP, Marques MEA, Marques SA. Mycosis fungoides and Sézary syndrome: clinical, histophathological and immunohistochemical review and update. An Bras Dermatol. 2012;87(6):817-30. doi: 10.1590/S0365-05962012000600001.

4. Kim YH, Liu HL, Mraz-Gernhard S, Varghese A, Hoppe RT. Long-term outcome of 525 patients with mycosis fungoides and Sezary syndrome: clinical prognostic factors and risk for disease progression. Arch Dermatol. 2003;139(7):85766. doi: 10.1001/archderm.139.7.857.

5. Brandford PT, Devessa SS, Anderson WF, Toro JR. Cutaneous Lymphoma incidence patterns in the United States: a population-base study of 3884 com equipe multidisciplinar envolvendo dermatologista, hematologista e laboratório clínico, sendo essencial o reconhecimento precoce da doença.

Conflitos de interesse: Os autores declaram que não existem conflitos de interesse. cases. Blood. 2009;113(21):5064-73. doi: 10.1182/ blood-2008-10-184168.

6. Sanches Jr JA, Moricz CZM, Neto CF. Processos linfoproliferativos da pele. Parte 2 - Linfomas cutâneos de células T e de células NK. An Bras Dermatol. 2006;81(1):725. doi: 10.1590/S0365-05962006000100002.

7. Prince MH, Whittakker S, Hoppe. How I treat mycosis fungoides and Sézary syndrome. Blood. 2009;114(20):433751. doi: 10.1182/blood-2009-07-202895.

8. Nashan D, Faulhaber D, Ständer S, Luger TA, Stadler R. Mycosis fungoides: a dermatologic masquerander. Br J Dermatol. 2007;156(1):1-10. doi: 10.1111/j.13652133.2006.07526.x.

9. Vonderheid EC, Bernengo MG, Burg G, Duvic M, Heald P, Laroche L, Olsen E, Pittelkow M, Russell-Jones R, Takigawa M, Willemze R; ISCL. Update on erythrodermic cutaneous T-cell lymphoma: report of the International Society for Cutaneous Lymphoma. J Am Acad Dermatol. 2002;46(1):95-106. doi: 10.1067/mjd.2002.118538.

10. Van Doorn R, Van Haselen CW, Van Voorst Vader PC, Geerts ML, Heule F, De Rie M, Steijlen PM, Dekker SK, Van Vloten WA, Willemze R. Mycosis fungoides: disease evolution and prognosis of 309 Dutch patients. Arch Dermatol. 2000;136 (4):504-10. doi: 10.1001/ archderm.136.4.504. 